\title{
Searching for simple rules in Pseudomonas aeruginosa biofilm formation
}

\author{
William Deveaux ${ }^{1}$ and Kumar Selvarajoo ${ }^{2^{*}}$ (1)
}

\begin{abstract}
Objective: Living cells display complex and non-linear behaviors, especially when posed to environmental threats. Here, to understand the self-organizing cooperative behavior of a microorganism Pseudomonas aeruginosa, we developed a discrete spatiotemporal cellular automata model based on simple physical rules, similar to Conway's game of life.

Results: The time evolution model simulations were experimentally verified for P. aeruginosa biofilm for both control and antibiotic azithromycin (AZM) treated condition. Our model suggests that AZM regulates the single cell motility, thereby resulting in delayed, but not abolished, biofilm formation. In addition, the model highlights the importance of reproduction by cell to cell interaction is key for biofilm formation. Overall, this work highlights another example where biological evolutionary complexity may be interpreted using rules taken from theoretical disciplines.
\end{abstract}

Keywords: Pseudomonas aeruginosa, Azithromycin, Computational model, Cellular automata, Self-organization

\section{Introduction}

Under external stress, microorganisms, such as bacteria and fungi, are able to produce cooperative response by forming biofilm. Biofilm are aggregates of cells, often produced through quorum sensing mechanisms or due to the secretion of extracellular polymeric substance [1]. They often pose problems to food and water safety. On the other hand, biofilm caused by pathogenic agents in human often show drug resistance [2]. Thus, each year, billions of dollars are at risk due to biofilm-mediated damage [3]. Despite the immense research over decades using low to high throughput experimental methodologies, the progress in understanding the regulatory mechanisms or controlling the progression of biofilm is highly limited. Therefore, better knowledge in biofilm growth and evolution is necessary, and scientists could explore interdisciplinary strengths to fill the missing gaps.

The pathogenic Gram-negative bacteria P. aeruginosa, is well-known for its intrinsic and acquired antibiotic resistance. It facilitates chronic infections in human by

\footnotetext{
*Correspondence: Kumar_selvarajoo@biotrans.a-star.edu.sg

2 Biotransformation Innovation Platform (BioTrans), Agency for Science,

Technology and Research A*STAR, Biopolis, Singapore

Full list of author information is available at the end of the article
}

its rapid ability to form biofilms [4]. Most previous works have mainly studied $P$. aeruginosa antibiotic resistance in their planktonic or single cell cultures. However, P. aeruginosa infection quickly spreads and form biofilm in certain diseases such as in the cystic fibrosis of the lung [5]. Thus, when infected in human, $P$. aeruginosa can cause death, especially for patients with cystic fibrosis, as they form biofilm that are resistant to current multi-drug antibiotic regimens [6]. Hence, the species and its biofilm are of considerable importance to medical care and patients' well-being.

In this paper, we studied the self-organization of planktonic single cell stage to cooperative biofilm stage of P. aeruginosa. For understanding self-organization, a large number of theoretical and computational works uses continuous differential equation approaches $[7,8]$, where the models require detailed mechanistic parameter values that are difficult to obtain from living systems. To overcome this limitation, here we utilized a discrete spatiotemporal computational methodology or cellular automata (CA), to predict the growth evolution mechanisms of $P$. aeruginosa [9]. CA models adopt simple physical rules or differential equations that matches experimental observation. By doing so, one can estimate 
the governing process or rules underlying the proliferation or cooperative behaviors.

Although there have been several efforts to model biofilm growth using CA in general $[10,11]$, here we focused on simple rules to specifically test 2-D spatio-temporal dynamics of $P$. aeruginosa biofilm in untreated (control) and antibiotic treated conditions.

\section{Main text}

\section{Data}

For experimental observation, we obtained time-series growth profiles of $P$. aeruginosa performed by Gillis and Iglewski [6], see Fig. 1. P. aeruginosa PAO1 biofilms were grown into flow cells with confocal microscopy over an extended time frame $[12,13]$. FAB medium amended with $20 \mu \mathrm{M} \mathrm{KNO}{ }_{3}$ [14], and treated with and without sub-MIC of AZM $(2 \mu \mathrm{g} / \mathrm{ml})$ were used for all flow cell studies.

Here, data for 2 conditions are shown; (a) without (wildtype, WT) and (b) with $2 \mu \mathrm{g} / \mathrm{ml}$ of azithromycin (or AZM, a macrolide antibiotic, WT+AZM). We observe that untreated $P$. aeruginosa rapidly forms clustered biofilm within $72 \mathrm{~h}$. The treated cells, although display

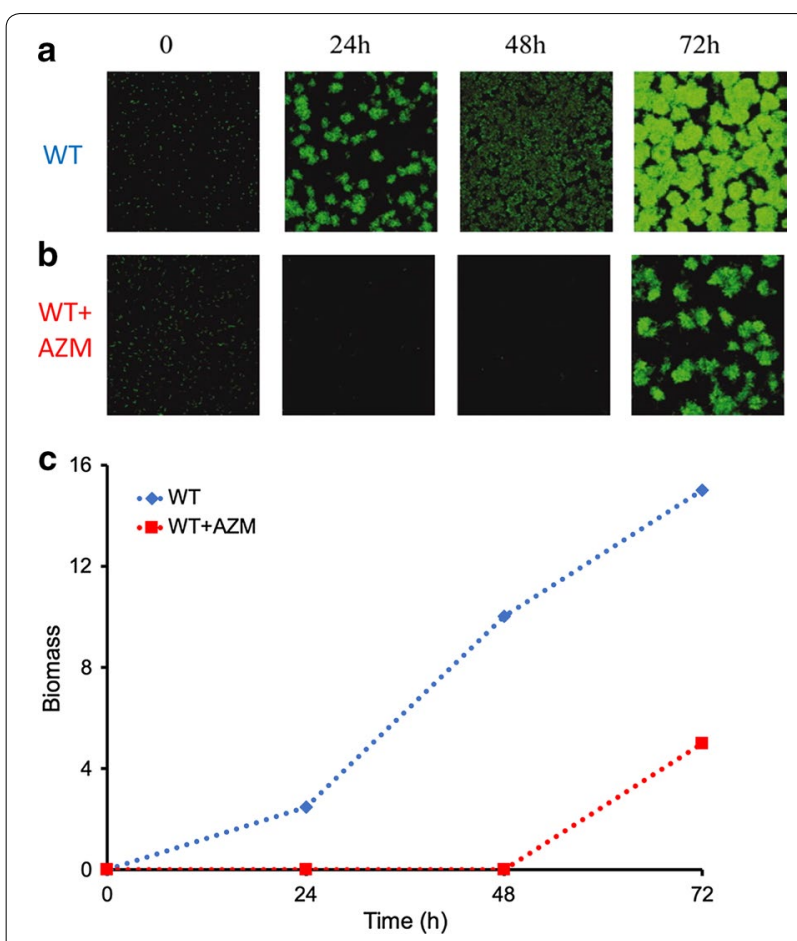

Fig. 1 Time series confocal microscopy of P. aeruginosa PAO1 colony, $\mathbf{a}$ without and $\mathbf{b}$ with antibiotic (azithromycin or AZM) treatment. $\mathbf{c}$ Total biomass $\left(\mu \mathrm{m}^{2} / \mu \mathrm{m}^{3}\right)$ in time. The profiles represent the average response of 5 independent experiments, their standard deviation is presented in the original experimental plot [6]. Biomass will be used as a proxy for cell numbers for simulations (Figures modified from [6], and permission to reproduce obtained) biofilm emergence at $72 \mathrm{~h}$, shows a much slower formation time.

\section{Methods}

Previously, to study cancer cell proliferation in control and drug treated conditions, we developed a discrete spatiotemporal CA model based on simple rules modified from Conway's game of life [14, 15]. The model's rules were iteratively guessed and modified until the simulations matched experimental observations [15]. The resultant rules were used to infer the proliferation properties of the control and treated cancer cells. Here, we extended the model to predict the biofilm formation of $P$. aeruginosa.

\section{Spatial temporal cellular automata Cellular automata model}

A 3-D CA model was developed in Matlab code consisting of $400 \times 400 \times 4$ cubic $(640,000)$ cells, with each cell having maximum 17 neighbors for the top and bottom planes, while 26 neighbors for other planes. The 8 corners, however, have a maximum of 7 neighbors, and 11 neighbors on the edges. We choose the empty initial cells large enough to avoid reaching the edges/corners within the simulated time steps. Note that our z-axis is small as the cells were originally cultured in 2-D flow cells plates, where cells often limited to a few layers on the vertical axis.

At time $=0 \mathrm{~h}$, for each condition, the cells were populated with live cells in random orientation that filled the spaces similar to Fig. 1. We found 5000 cells (Fig. 2a, leftmost panel) were distributed in a way that was similar to actual cell distribution in Fig. 1. The CA rules (see "Cellular automata model rules" section below) were applied from time step 1 onwards.

\section{Cellular automata model rules}

Our previous cancer CA model had rules that were modified from Conway's game of life. The rules, although abstract or oversimplification, can generate complex self-organizing spatiotemporal patterns that have been explored in numerous scientific fields. Our intention here is to first use these popular simple rules and gradually modify them to find a suitable set of rules for $P$. aeruginosa.

We adopted a similar approach where we began the model with rules:

i. Any immotile cell with less than $X_{1}$ live neighbors dies, caused by under-population.

ii. Any immotile cell with $X_{2}$ or $X_{3}$ live neighbors becomes motile cell on to the next generation. 


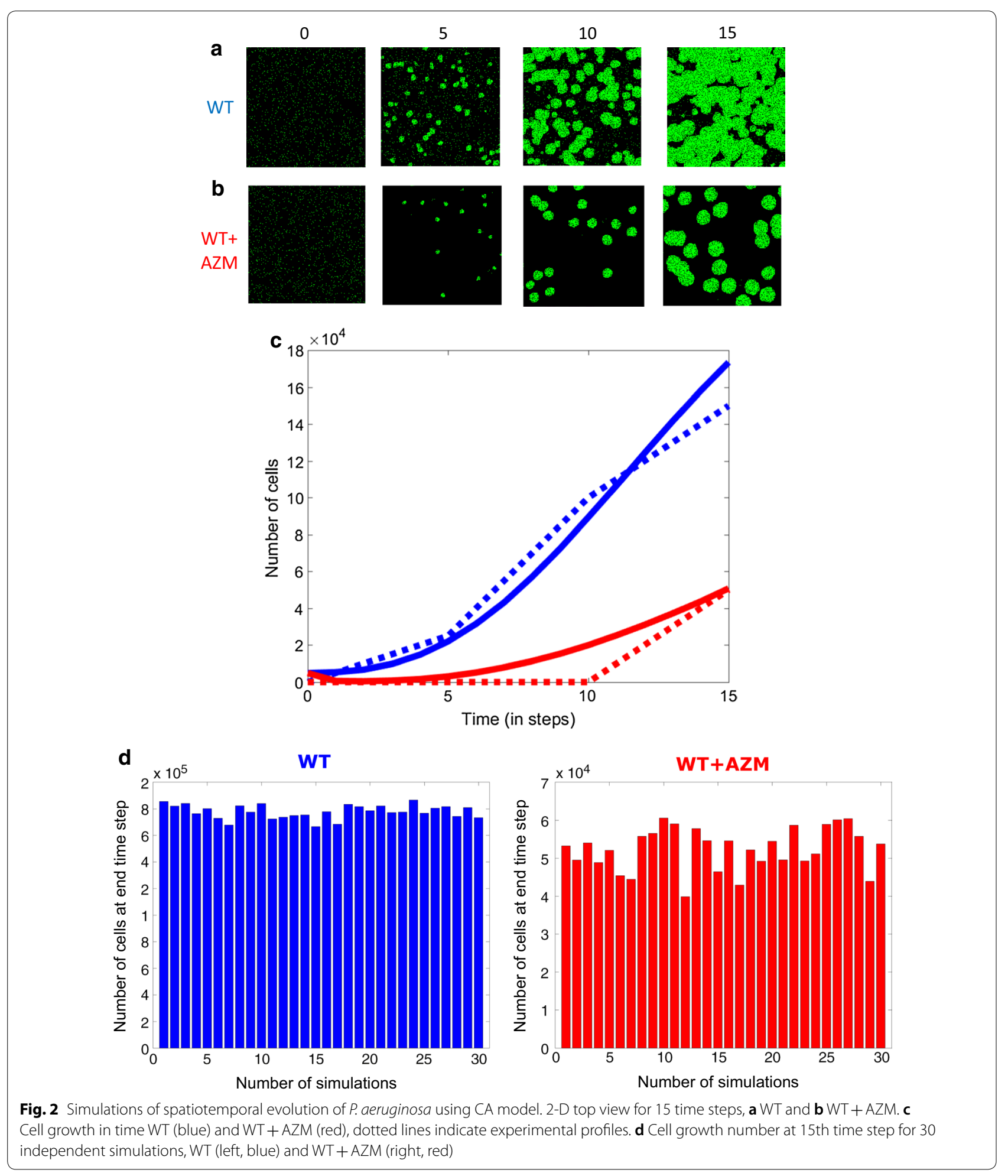


iii. Any immotile cell with more than $X_{4}$ neighbors dies, caused by overcrowding.

iv. Any dead/empty cell with $X_{5}$ to $X_{6}$ live neighbors becomes live cell as by reproduction (division).

v. Any motile cell moves randomly to another empty space in time.

vi. Any motile cell that cannot move becomes immotile cell on the next generation.

where for Conway's game of life, $X_{1}=2, X_{2}=2, X_{3}=3$, $X_{4}=3, X_{5}=3, X_{6}=3$. Here we will fit $X_{1}$ to $X_{6}$ using genetic algorithm with the experimental growth numbers in Fig. 1. Note that we have introduced additional rules 5 and 6 to consider movement of single cells in time, since planktonic single $P$. aeruginosa contain polar flagellum which allows them to be motile. We also introduced a new parameter for the percentage of motile cells.

\section{Results}

Figure $2 \mathrm{a}-\mathrm{c}$ shows the simulations of our CA model fitted to $P$. aeruginosa growth in Fig. 1 . Basically, we were required to fit $X_{1}$ to $X_{6}$ separately for the WT and $\mathrm{WT}+\mathrm{AZM}$ condition. We performed hundreds of simulations, using the aid of genetic algorithm to fit the data [15]. Notably, the model parameters remained the same as Conway's game of life. However, the main difference between the 2 models pointed to only one key parameter: the percentage of moving or motile cells (Additional file 1: Table S1). In other words, according to our simulations, cell movement is repressed by the antibiotic AZM resulting in slower growth rates.

We also simulated the final outcome for 30 independent simulations, to check the effect of variability due to random orientation of initial cell distributions. Figure 2D shows the random positioning of cells on average supports the overall experiments, and the final cell numbers do not show any significant variation between the runs.

Using the WT and WT + AZM fitted models, we subsequently simulated the longer term effect on the cell numbers. Notably, after 60 time steps, both model converges to the same cell numbers (Fig. 3a). This result is reminiscent of the growth curves shown by another work by Häussler et al. [16], who used the same protocol as Gillis and Iglewski [6].

Next, we investigated, from the rules, which one is key for suppressing the resurgence of cell proliferation and biofilm formation. After several considerations, we found that rule 4 parameters are crucial for suppressing cell proliferation (Fig. 3b). In other words, our model proposes the development of drugs that would be able to regulate the dispersion of cells or that prevents cell cluttering. That is, according to rule 4 , more empty cells
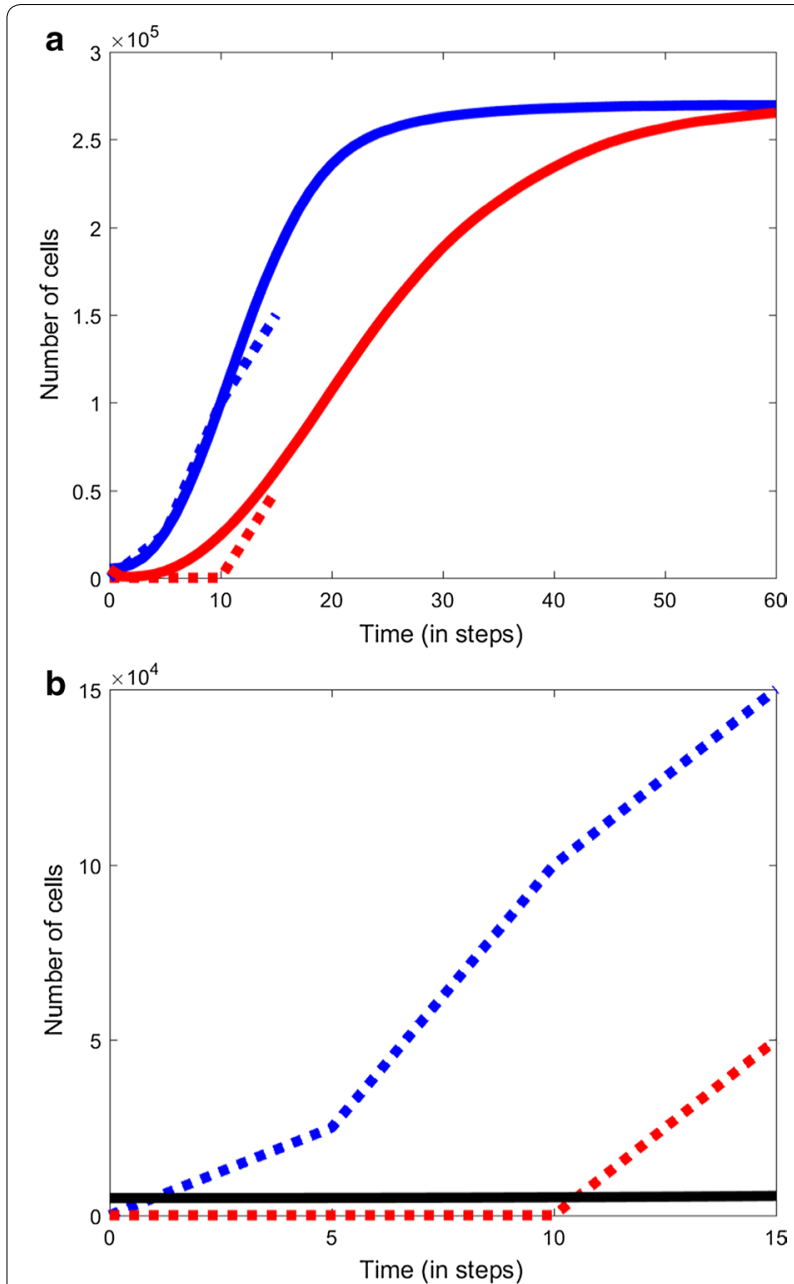

Fig. 3 Simulations of spatiotemporal evolution of $P$. aeruginosa. a Longer term CA model simulations using WT (blue) and WT + AZM (red), $\mathbf{b}$ modified CA model simulations (rule $4, X_{5}=5$ and $X_{6}=6$ ) that prevents biofilm formation

between cells prevent cell to cell contact, thereby, is crucial for controlling biofilm formation.

\section{Discussion}

In this paper, we have developed a discrete CA model to understand the spatiotemporal self-organizing patterns of $P$. aeruginosa biofilm. The initial rules were taken directly from the famous Conway's game of life with two additional rules included to factor single cell flagellar random movement. The parameters of the model were fitted with experimental profiles available for the biofilm growth for two conditions. As a result, we developed a single CA model, with only one parameter (\% of motile cells) separating the WT and $\mathrm{WT}+\mathrm{AZM}$ simulations. 
Notably, our model simulations not only recapitulate the growth profiles of both the untreated and treated biofilm successfully (Figs. 1c and 2c), they also capture the spatial organization of the cells/biofilm over time (Figs. 1a, b and 2a, b). The model predictions suggest that adding the antibiotic agent inhibits the movement of certain single planktonic P. aeruginosa which retards their growth. However, subsequently, the inhibition succumbs due to the other rules ( 1 and 4 ) to form delayed biofilm. Thus, our model predictions indicate that AZM, on top of regulating bacterial quorum sensing mechanism and metabolism, is also regulating the cell movement mechanisms such as those involved in flagellar functioning. This delays the overall biofilm progression.

Experimentally, although AZM is shown to suppress $P$. aeruginosa biofilm $[6,16]$, its mechanism of action still remains poorly understood. We next searched the literature on high-throughput transcriptomics and proteomics related works on AZM treated P. aeruginosa. Remarkably, we found the work by Häussler et al. [16] supporting our model prediction. In their work, they have shown that the genes and proteins related to flagellar are indeed down-regulated using the same dosage of AZM treatment compared with WT.

Moving further, to find a condition that would effectively suppress $P$. aeruginosa biofilm formation, we searched for the most appropriate rules and their parameter values. The best model suggests that rule 4 should have parameter values $X_{5}=5$ and $X_{6}=6$, which will prevent biofilm formation and keep the cell numbers almost unchanged throughout time (Fig. 3b). It will be interesting and crucial to identify the biological target that will regulate rule 4 by preventing cell to cell contact. Trying adhesins inhibitors with AZM may be a viable next option. Another option is to check the application of biofilm quorum-sensing regulators, such as catechin. Although previous works have shown their positive effect in biofilm regulation, their actual mechanism on the cell to cell contact remains elusive $[17,18]$.

In summary, our work here highlights the need for interdisciplinary research to understand and combat the complexities of living systems, such as controlling the pathogenic microorganisms that endanger the lives of infected people.

\section{Limitations}

Further work is required to experimentally validate our final model prediction, such as trying the co-application of adhesins inhibitors or catechin with AZM. Also, the model should be expanded to include the simulation and testing of multi-species quorum-sensing bacteria evolution, which is usually a major concern in chronic infection.

\section{Supplementary information}

Supplementary information accompanies this paper at https://doi. org/10.1186/s13104-019-4795-x.

Additional file 1: Table S1. CA model parameters for fitting WT and WT+AZM.

\section{Abbreviations}

CA: cellular automata; AZM: azithromycin; WT: wildtype; FAB: fastidious anaerobe broth; $\mathrm{KNO}_{3}$ : potassium nitrate; MIC: minimum inhibitory concentration.

\section{Acknowledgements}

N. Lindley and Y. Kanagasundram for discussion.

\section{Authors' contributions}

WD constructed the model and performed simulations. KS conceived the idea and rules, supervised the work and wrote the paper. All authors read and approved the final manuscript.

\section{Funding}

The authors thank BioTrans for funding (IAF-PP) the modeling work. The funder played no role in study design; collection, analysis, and interpretation of data; writing of the report; or in the decision to submit the article for publication

\section{Availability of data and materials}

The CA model with user instructions is found on URL: https://github.com/Eclio n/cell-automata

\section{Ethics approval and consent to participate}

Not applicable.

\section{Consent for publication}

Not applicable.

\section{Competing interests}

The authors declare that they have no competing interests.

\section{Author details}

${ }^{1}$ École nationale d'ingénieurs de Brest, Brest, France. ${ }^{2}$ Biotransformation Innovation Platform (BioTrans), Agency for Science, Technology and Research A*STAR, Biopolis, Singapore.

Received: 17 October 2019 Accepted: 11 November 2019

Published online: 21 November 2019

References

1. Flemming $\mathrm{HC}$, et al. Biofilms: an emergent form of bacterial life. Nat Rev Microbiol. 2016;14:563-75.

2. Piras V, Chiow A, Selvarajoo K. Long-range order and short-range disorder in Saccharomyces cerevisiae biofilm. Eng Biol. 2019:3:12-9.

3. Worthington RJ, Richards JJ, Melander C. Small molecule control of bacterial biofilms. Org Biomol Chem. 2012:10:7457-74.

4. Ahmed MN, Porse A, Sommer MOA, Høiby N, Ciofu O. Evolution of antibiotic resistance in biofilm and planktonic Pseudomonas aeruginosa populations exposed to subinhibitory levels of ciprofloxacin. Antimicrob Agents Chemother. 2018;62:e00320-18.

5. de Jong PA, et al. Progressive damage on high resolution computed tomography despite stable lung function in cystic fibrosis. Eur Respir J. 2004:23:93-7.

6. Gillis RJ, Iglewski BH. Azithromycin retards Pseudomonas aeruginosa biofilm formation. J Clin Microbiol. 2004:42:5842-5. 
7. Maini PK, Woolley TE, Baker RE, Gaffney EA, Lee SS. Turing's model for biological pattern formation and the robustness problem. Int Focus. 2012;2:487-96.

8. Selvarajoo K. Complexity of biochemical and genetic responses reduced using simple theoretical models. Methods Mol Biol. 2018;1702:171-201.

9. Wolfram S. Statistical mechanics of cellular automata. Rev Mod Phys. 1983;55:601-44.

10. Skoneczny S. Cellular automata-based modelling and simulation of biofilm structure on multi-core computers. Water Sci Technol. 2015;72:2071-81.

11. Pizarro GE, García C, Moreno R, Sepúlveda ME. Two-dimensional cellular automaton model for mixed-culture biofilm. Water Sci Technol. 2004;49:193-8.

12. De Kievit TR, Gillis R, Marx S, Brown C, Iglewski BH. Quorum-sensing genes in Pseudomonas aeruginosa biofilms: their role and expression patterns. Appl Environ Microbiol. 2001;67:1865-73.

13. Heydorn A, et al. Experimental reproducibility in flow-chamber biofilms. Microbiology. 2000;146:2409-15.

14. Gardner M. Mathematical games: the fantastic combinations of John Conway's new solitaire game"life". Sci Am. 1970;223:120-3.
15. Deveaux W, Hayashi K, Selvarajoo K. Defining rules for cancer cell proliferation in TRAIL stimulation. NPJ Syst Biol Appl. 2019;5:5.

16. Nalca Y, et al. Quorum-sensing antagonistic activities of azithromycin in Pseudomonas aeruginosa PAO1: a global approach. Antimicrob Agents Chemother. 2006;50:1680-8.

17. Vandeputte $\mathrm{OM}$, et al. Identification of catechin as one of the flavonoids from Combretum albiflorum bark extract that reduces the production of quorum-sensing-controlled virulence factors in Pseudomonas aeruginosa PAO1. Appl Environ Microbiol. 2010;76:243-53.

18. Matsunaga T, et al. The inhibitory effects of catechins on biofilm formation by the periodontopathogenic bacterium, Eikenella corrodens. Biosci Biotechnol Biochem. 2010;74:2445-50.

\section{Publisher's Note}

Springer Nature remains neutral with regard to jurisdictional claims in published maps and institutional affiliations.
Ready to submit your research? Choose BMC and benefit from:

- fast, convenient online submission

- thorough peer review by experienced researchers in your field

- rapid publication on acceptance

- support for research data, including large and complex data types

- gold Open Access which fosters wider collaboration and increased citations

- maximum visibility for your research: over $100 \mathrm{M}$ website views per year

At BMC, research is always in progress.

Learn more biomedcentral.com/submissions 